TITRE: L'ACCOMPAGNEMENT DES PREMIÈRES ÉCRITURES : EFFETS ET PRATIQUES - UNE SYNTHÈSE

Auteur(s): LoḮ Pulido et MARIe-France Morin, CREALEC, FACulté d’ÉducATIOn, Université de SHERBROOKE (QuÉBEC, CANADA)

Publication: Perspectives actuelles sur L'APprentissage de LA LECTURE ET de L'ÉCRITURE/ CONTRIBUTIONS ABOUT LEARNING TO READ AND WRITE - ACTES DU SYMPOSIUM INTERNATIONAL SUR LA LITÉRACIE À L'ÉCOLE/INTERNATIONAL SYMPOSIUM FOR EDUCATIONAL LITERACY (SILE/ISEL) 2015

PAGES: $57-79$

Directeurs: Marie-France Morin, Denis Alamargot et Carolina Gonçalves.

ÉditeUR: LES ÉdITIONS DE L'UNIVERSITÉ DE SHERBROOKE, 2016.

ISBN: 978-2-7622-0355-4

URI: HTTP://HDL.HANDLE.NET/11143/10229

DOI: HTTPS://DOI.ORG/10.17118/11143/10229 


\section{L’accompagnement des premières écritures : effets et pratiques - une synthèse}

Loïc Pulido, Université du Québec à Chicoutimi

Marie-France Morin, CREALEC, Faculté d'éducation, Université de Sherbrooke (Québec, Canada)

Résumé : De nombreuses recherches publiées ces dernières décennies portent sur l'accompagnement, à l'école maternelle, des premières tentatives d'écriture des enfants. Cette contribution vise à réaliser une synthèse des études sur ce thème, articulée autour de deux principales questions: 1) que sait-on de l'effet de l'accompagnement des premières écritures des élèves? Des recherches expérimentales ou quasi expérimentales permettent d'aboutir à l'idée d'un impact généralement positif de ce genre de pratiques sur le développement des premières habiletés en littéracie en maternelle et lors de la première année du primaire. 2) Que sait-on de la manière dont on peut accompagner les premières écritures des élèves de maternelle? Des recherches proposant des réflexions étayées sur la manière de s'y prendre et d'autres s'attachant à décrire les pratiques d'enseignants qui mettent en place ces accompagnements seront mobilisées pour répondre à cette question. L'agglomération des réponses à ces deux questions permettra de mieux cerner l'intérêt d'accompagner les premières écritures des enfants de maternelle.

Mots clés : Accompagnement, éveil à l'écrit, écritures inventées, maternelle

Abstract: Many studies published during these last decades deal with the support of the first attempts the children make in writing, in kindergarten. This contribution targets the realization of a synthesis of studies on this theme, revolving around two main questions: 1) What do we know about the effects of the support given to the first written productions? Some experimental and quasi-experimental studies lead to the idea that the impact of this writing support is generally positive on the development of the first literacy skills in kindergarten and during the first years of primary school. 2) What do we know about the way this support can be given to the children in kindergarten? Studies offering a reflection on how to do it, and others describing the experiences of teachers that implemented this support, will be gathered to answer this question.

The gathering of the answers to these two questions will allow us to grasp the interest of supporting children in their first written work in kindergarten.

Key words: Support, early learning, invented spelling, kindergarten. 


\section{Introduction}

Il est un âge auquel les enfants s'intéressent à l'écriture: ils tentent d'imiter l'écriture adoptée par les adultes qui les entourent, observent attentivement les écrits qu'ils rencontrent, se posent et posent des questions à leur propos. Dès les années 1970-1980, des chercheuses comme Carol Read (1971) ou Emilia Ferreiro et Ana Teberosky (1982) ont montré que ces comportements n'étaient pas anodins ou accidentels et qu'ils traduisaient l'entrée de l'enfant dans une démarche de construction de connaissances sur le fonctionnement de la langue écrite. À partir de ces travaux fondateurs, plusieurs chercheurs ont étudié les premières tentatives d'écriture des jeunes enfants qui n'avaient pas encore été soumis à un enseignement formel de la langue écrite. Ces études, qui ont été menées auprès d'enfants de différents pays et de différentes langues, ont confirmé les premiers travaux en soutenant fortement l'idée que les jeunes enfants de maternelle ont commencé à construire des connaissances variées sur la langue écrite et adoptent des procédures qui les soutiendront dans l'appropriation de comportements à l'écrit plus conformes aux conventions qui régissent l'écriture (Alves Martins, Silva et Pereira, 2010; Fraquet et David, 2013; Montésinos-Gelet et Besse, 2003; Morin, 2007a; Noyer-Martin et Baldy, 2008; Pasa, Creuzet et Fijalkow, 2006; Treiman, Decker, Kessler et Pollo, 2015).

Près de cinquante années plus tard, les enseignants sont explicitement encouragés à soutenir les premières tentatives d'écriture. Par exemple, dans le contexte francophone, les enseignants québécois sont encouragés à faire en sorte que les élèves de maternelle explorent leurs écritures spontanées (MELS, 2001). Les enseignants français sont, de façon similaire, incités à faire en sorte que les élèves produisent des écrits dans un esprit de découverte de leur fonctionnement (MEN, 2015). Ce contexte appelle deux questions qui seront abordées dans ce chapitre. La première : quel est l'effet de ce genre d'accompagnement? Depuis 2005 environ, plusieurs recherches se sont intéressées à cette question. La seconde: que sait-on de la manière dont on peut accompagner les premières écritures à l'école? Des équipes de recherche ont élaboré, depuis les années 2000, des préconisations et/ou ont analysé comment des enseignants se les appropriaient. Leurs travaux seront utilisés pour éclairer cette question. L'agglomération des éléments de réponses à ces deux questions apportera des arguments en faveur de l'intérêt à accompagner les premières écritures des enfants, à l'école maternelle, et offrira une synthèse des résultats de recherches qui peuvent servir d'appui au développement des pratiques des enseignants. 


\section{Quel est l'effet de l'accompagnement des premières écritures des enfants de maternelle?}

Si les enseignants de maternelle sont encouragés à faire écrire leurs élèves, c'est parce que des données de recherche congruentes attestent d'un effet positif de ce genre d'accompagnement. L'objectif de cette section est d'offrir une synthèse permettant de mieux cerner la nature de ces effets. Lorsque des chercheurs souhaitent prendre la mesure des effets de dispositifs pédagogiques avec de fortes implications didactiques, ils recourent à des méthodologies de type expérimental ou quasi expérimental. Ils comparent les progrès d'élèves placés dans différentes conditions correspondant aux dispositifs dont ils souhaitent mesurer les effets à ceux d'élèves placés dans une condition contrôle. Quatorze recherches publiées à ce jour et accessibles par les bases de données de psychologie ou de sciences de l'éducation francophones et internationales anglophones portent sur la question de l'effet de l'accompagnement des premières écritures des enfants à l'école maternelle. Ces recherches et leurs principales caractéristiques sont présentées dans le tableau 1.

Tableau 1 Les recherches portant sur l'effet de l'accompagnement des premières écritures des enfants et leurs principales caractéristiques

\begin{tabular}{|c|c|c|c|}
\hline Études empiriques recensées & $\begin{array}{l}\text { Nombre de } \\
\text { participants }\end{array}$ & Durée de l'intervention & Conditions expérimentales \\
\hline Alves Martins et Silva (2006) & 90 & 8 sessions de travail & $\begin{array}{l}\text { Accompagnement des premières écritures vs activités } \\
\text { avec des blocs }\end{array}$ \\
\hline $\begin{array}{l}\text { Alves Martins, Albuquerque, Salvador } \\
\text { et Silva (2013) }\end{array}$ & 108 & $\begin{array}{l}10 \text { sessions de } 15 \text { minutes } \\
\text { sur } 5 \text { semaines }\end{array}$ & Accompagnement des premières écritures vs dessin \\
\hline $\begin{array}{l}\text { Alves Martins, Salvador, Albuquerque } \\
\text { et Silva (2016) }\end{array}$ & 160 & $\begin{array}{l}10 \text { sessions de } 15 \text { minutes } \\
\text { sur } 5 \text { semaines }\end{array}$ & $\begin{array}{l}\text { Accompagnement des premières écritures avec interac } \\
\text { tions entre élèves vs sans interaction vs dessin }\end{array}$ \\
\hline $\begin{array}{l}\text { Hofslundsengen, Hagtvet et Gustafs- } \\
\text { son (2016) }\end{array}$ & 105 & $\begin{array}{l}4 \text { sessions de } 20 \text { minutes } \\
\text { par semaine sur } 4 \\
\text { semaines }\end{array}$ & $\begin{array}{l}\text { Accompagnement des tentatives d'écriture vs pratiques } \\
\text { de classe habituelles }\end{array}$ \\
\hline Levin et Aram (2013) & 197 & $\begin{array}{l}2 \text { sessions individuelles } \\
\text { sur } 16 \text { semaines }\end{array}$ & $\begin{array}{l}\text { Accompagnement des premières écritures vs pratiques } \\
\text { de classe habituelles }\end{array}$ \\
\hline
\end{tabular}




\begin{tabular}{|c|c|c|c|}
\hline Études empiriques recensées & $\begin{array}{l}\text { Nombre de } \\
\text { participants }\end{array}$ & Durée de l'intervention & Conditions expérimentales \\
\hline Montésinos-Gelet et Morin (2005) & 20 & $\begin{array}{l}\text { Travail au quotidien, en } \\
\text { classe, pendant l'année } \\
\text { scolaire }\end{array}$ & Accompagnement vs non accompagnement \\
\hline Morin et Montésinos-Gelet (2007) & $\begin{array}{l}90 \\
\text { (enfants à } \\
\text { risque) }\end{array}$ & $\begin{array}{l}\text { Travail quotidien en } \\
\text { classe, pendant } 1 \text { an }\end{array}$ & Accompagnement vs non accompagnement \\
\hline O'connor et Jenkins (1995) & $\begin{array}{l}10 \\
\text { (enfants avec } \\
\text { retard de déve- } \\
\text { loppe-ment) }\end{array}$ & $\begin{array}{l}20 \text { sessions de } 10 \text { minutes } \\
\text { sur } 4 \text { semaines }\end{array}$ & $\begin{array}{l}\text { Accompagnement des premières écritures vs écoute } \\
\text { d'histoires lues }\end{array}$ \\
\hline Ouellette et Sénéchal (2008) & 46 & $\begin{array}{l}9 \text { sessions de } 25 \text { minutes } \\
\text { sur } 4 \text { semaines }\end{array}$ & Accompagnement des premières écritures vs dessin. \\
\hline Ouellette, Sénéchal et Haley (2013) & $\begin{array}{l}40 \\
\text { (enfants stimulés } \\
\text { au plan de la } \\
\text { littéracie) }\end{array}$ & $\begin{array}{l}16 \text { sessions de } 20 \text { minutes } \\
\text { sur } 8 \text { semaines }\end{array}$ & $\begin{array}{l}\text { Accompagnement des premières écritures vs entraine- } \\
\text { ment phonologique }\end{array}$ \\
\hline $\begin{array}{l}\text { Rieben, Ntamakiliro, Gonthier et Fayol } \\
\text { (2005) }\end{array}$ & 148 & $\begin{array}{l}18 \text { sessions de } 20 \text { mi- } \\
\text { nutes, sur } 6 \text { mois }\end{array}$ & $\begin{array}{l}\text { Accompagnement des premières écritures vs production } \\
\text { de tentatives d'écriture sans feedback vs copie de mots } \\
\text { vs dessin }\end{array}$ \\
\hline Santoro, Coyne et Simmons (2008) & $\begin{array}{l}116 \\
\text { (enfants risquant } \\
\text { d'avoir des } \\
\text { difficultés à ap- } \\
\text { prendre à lire) }\end{array}$ & $\begin{array}{l}108 \text { sessions de } 30 \text { mi- } \\
\text { nutes, sur } 7 \text { mois }\end{array}$ & $\begin{array}{l}\text { Entrainement phonologique + accompagnement des } \\
\text { premières écritures vs entrainement phonologique + } \\
\text { écoute d'histoires lues }\end{array}$ \\
\hline $\begin{array}{l}\text { Sénéchal, Ouellette, Pagan et Lever } \\
\text { (2012) }\end{array}$ & $\begin{array}{l}56 \\
\text { (enfants ayant de } \\
\text { faibles habiletés } \\
\text { phonolo-giques) }\end{array}$ & $\begin{array}{l}16 \text { sessions de } 20 \text { minutes } \\
\text { sur } 8 \text { semaines }\end{array}$ & $\begin{array}{l}\text { Accompagnement des premières écritures vs entraine- } \\
\text { ment phonologique vs lecture dialoguée }\end{array}$ \\
\hline $\begin{array}{l}\text { Vasconcelos Horta et Alves Martins } \\
\text { (2011) }\end{array}$ & 56 & $\begin{array}{l}4 \text { sessions de } 15 \text { minutes } \\
\text { sur } 4 \text { semaines }\end{array}$ & $\begin{array}{l}\text { Accompagnement de tentatives d'écriture de mots } \\
\text { contenant les sons f et s vs accompagnement des } \\
\text { tentatives d'écritures de mots contenant les sons p et t } \\
\text { vs activités de blocs }\end{array}$ \\
\hline
\end{tabular}

Les recherches recensées ont été réalisées dans différents contextes linguistiques. Plus précisément, certaines études ont été menées auprès de jeunes scripteurs apprenant un système orthographique plus ou moins opaque (études menées auprès d'enfants lusophones: Alves Martins et al. 2006 ; 2013 ; 2014 ; études menées auprès d'enfants anglophones: O'Connor et Jenkins, 1995; Ouellette et al., 2008 et 2013; Sénéchal et al., 2012; études menées auprès d'enfants francophones : Rieben et al., 
2005; Montésinos-Gelet et Morin, 2005; Morin et Montésinos-Gelet, 2007; auprès d'enfants israéliens: Levin et Aram, 2013; auprès d'enfants suédois : Hofslundsengen, Hagtvet et Gustafsson, 2016). À cet égard, des chercheurs ont déjà attiré l'attention sur le fait que les systèmes d'écriture plus réguliers ou transparents (Fayol et Jaffré, 2014) suscitaient moins de résistance chez les jeunes apprenants, en comparaison avec des systèmes orthographiques plus opaques, comme l'anglais ou le français.

Ces recherches proposent des formes d'accompagnement variées. En effet, certaines invitent les enfants à produire des mots en induisant une stratégie de segmentation et de recherche d'un graphème (O'Connor et Jenkins, 1995), alors que d'autres les invitent à réfléchir à l'écart entre leur écriture ou celle d'un pair et la norme orthographique (Montésinos-Gelet et Morin, 2005; Morin et Montésinos-Gelet, 2007; Rieben et al., 2005). D’autres études (Ouelette et Sénéchal, 2008; Alves Martins et al., 2006 et 2013) utilisent quant à elles un dispositif qui invite les enfants à réfléchir à l'écart existant entre leur écriture d'un mot et une écriture un peu plus élaborée sans pour autant correspondre à la norme (par exemple, si un enfant écrit le mot « kfé » pour café, on lui demande de réfléchir à ce qu'apporterait de l'écrire «kafé »). Enfin, dans leur étude, Hofslundsengen, Hagtvet et Gustafsson (2016) utilisent plusieurs de ces formes d'accompagnement en fonction de l'avancée des élèves dans leur cheminement.

Malgré ces différences quant à la nature du système écrit à apprendre et quant à la nature des dispositifs d'accompagnement sollicités, il ressort globalement de ces études que l'accompagnement des premières écritures aide les enfants à développer leurs compétences phonologiques, le degré de sophistication de leurs tentatives d'écriture et leurs premières capacités à lire des mots (décodage ou segmentation).

\section{Accompagnement des premières écritures et compétences phonologiques}

Tout d'abord, les résultats des études réalisées indiquent clairement que l'accompagnement des premières écritures a un impact positif sur les compétences phonologiques des enfants, c'est-à-dire sur leur capacité à segmenter les mots en syllabes ou en phonèmes à l'oral. Comme l'ont montré plusieurs études, ces compétences constituent un prédicteur d'un apprentissage aisé de la lecture (par exemple, Goswami et Bryant, 1990). En ce sens, il semble que l'accompagnement des premières écritures permet aux enfants de progresser dans différentes tâches habituellement utilisées pour évaluer les compétences phonologiques des enfants. Certaines études ont ainsi montré, qu'accompagnés, les enfants progressent dans des tâches d'identification de phonème; par exemple, apparier «bateau » et « bêche » si «bateau », « bêche » et « poulet» sont présentés (Rieben et al., 2005; Ouelette et al., 2008; 2013; Alves Martins et Silva, 2006; Sénéchal et al., 2012), ou encore dans des tâches de suppression du phonème initial d'un mot (par exemple, dire ce qu'il reste du mot manteau si on enlève le son du début, les enfants devant répondre anteau) (Alves Martins et Silva, 2006; Sénéchal et al., 2012). D’autres études ont également montré les effets positifs des situations d'écriture sur des habiletés phonologiques plus complexes comme la segmentation phonémique (par exemple, 
dire de quels petits sons est composé le mot « vélo ») (Alves Martins et Silva, 2006) et l'assemblage phonémique (par exemple, dire quel mot est composé des sons [m] [o] [t] [o]) (Sénéchal et al., 2012).

\section{Accompagnement des premières écritures et capacités d'encodage orthographique}

Les recherches réalisées sur les effets de l'accompagnement des premières écritures des enfants rapportent également une influence positive de celui-ci sur les capacités d'encodage à l'écrit de mots donnés à l'oral. Cela signifie concrètement que lorsque l'on accompagne les premières écritures des enfants, ils apprennent à mieux orthographier les mots qu'on leur dicte. Ces résultats maintes fois rapportés (Alves Martins et al., 2006; 2013; 2014; Vasconcelos Horta et al., 2011; Montésinos-Gelet et Morin, 2005; Sénéchal et al., 2012; O'Connor et al., 1996; Rieben et al., 2005) soulignent une progression pour l'encodage des segments de mots travaillés ou des segments de mots non travaillés et traduisent ainsi une meilleure appropriation du système écrit par les enfants.

\section{Accompagnement des premières écritures et capacités en lecture}

Outre des effets positifs sur le traitement de la langue orale et sur l'acquisition des premières capacités orthographiques, les études qui s'intéressent aux effets des accompagnements des premières écritures relèvent des bénéfices pour le développement des premières habiletés en lecture. Plusieurs recherches (Alves Martins et al., 2006; 2014; O'Connor et al., 1995; Rieben et al., 2005) montrent que les enfants accompagnés dans leurs premières écritures en maternelle semblent en effet apprendre mieux que les autres à décoder quelques mots ou portions de mots simples, pour peu qu'ils aient eu à les écrire lorsqu'ils étaient accompagnés. Certaines recherches montrent même un tel effet sur des portions de mots qui n'ont pas été écrites au préalable (Morin et Montésinos-Gelet, 2007; O'Connor et Jenkins, 1995), mais cet effet est moins robuste, puisque d'autres recherches qui l'ont étudié ne l'ont pas mis en avant (Sénéchal et al., 2012; Rieben et al., 2005). Peut-être est-ce lié à une difficulté de transfert. Quoi qu'il en soit, ensemble, ces résultats vont dans le sens d'une meilleure compréhension du principe alphabétique (c'est-à-dire d'une meilleure compréhension des relations entre les sons des mots à l'oral et les signes écrits) et d'une meilleure capacité à combiner les différentes unités de la syllabe.

\section{Synthèse : accompagner les premières écritures aide à entrer dans la littéracie}

Même si les études évoquées dans cette section ont été réalisées auprès de différentes populations et ont mobilisé différentes dispositions d'accompagnement, il ressort des effets positifs de l'accompagnement des premières écritures sur les compétences phonologiques, sur les capacités d'encodage et sur les premières capacités de déchiffrage en lecture des élèves. Accompagner les premières écritures des enfants à la maternelle peut ainsi être envisagé comme une voie pour faciliter les premiers moments d'apprentissages litéraciques. Comme ces effets ont également été relevés auprès 
d'enfants présentant des risques d'avoir des difficultés pour apprendre à lire, on peut considérer qu'accompagner les premières écritures est un bon moyen de prévenir les difficultés d'apprentissage de la lecture au primaire.

\section{Que sait-on de la manière dont on peut accompagner les premières écritures des enfants à l'école?}

L'intérêt de l'accompagnement des premières écritures étant avéré, se pose la question des modalités pratiques de cet accompagnement. Depuis une quinzaine d'années, des chercheurs s'intéressent à cette question. D'une part, leurs travaux permettent de dégager des principes généraux qui favoriseraient les découvertes que font les enfants grâce à leurs premières écritures. D’autre part, certains travaux proposent des démarches didactiques et des outils pour mettre en place ces démarches.

\section{Principes généraux pour que les enfants prennent du plaisir et apprennent en essayant d'écrire}

Quatre principes généraux semblent partagés par la plupart des travaux qui s'intéressent aux moyens de tirer parti des premières écritures des enfants en contexte scolaire.

Encourager les enfants à faire des tentatives d'écriture et à verbaliser leurs connaissances et stratégies

Mettre l'accent sur l'accompagnement des premières écritures des enfants, c'est s'inscrire dans un paradigme qui considère que le contexte dans lequel l'enfant est invité à écrire constitue un facteur important pour favoriser la progression de ce dernier à l'égard de sa compréhension de la langue écrite. Cette position ne va pas de soi. En effet, les premiers travaux sur les débuts de l'écriture, ceux déjà évoqués de Ferreiro notamment, se situaient dans une perspective constructiviste et mettaient donc au cœur de leur analyse l'activité de l'enfant. Pourtant, Fijalkow, Cussac-Pomel et Hannouz (2009), en passant en revue les travaux existant sur l'évolution des écrits des jeunes enfants, ont montré que les études qui ne prennent pas en compte certains éléments de contexte, comme les contextes linguistique et didactique par exemple, peinent à obtenir des résultats congruents concernant les découvertes des enfants. Dans leur analyse, Fijalkow et al. (2009) montrent, à partir d'un échantillon de quelques études réalisées en didactique expérimentale, qu'il existe un lien étroit entre les découvertes des enfants, ce qu'ils sont capables de dire de leur activité, et ce qui leur est suggéré par la ou par les personnes qui les accompagnent.

Ce que l'enfant est en mesure de dire et les conversations qui vont se mettre en place à partir de là seront donc déterminants pour que les premières écritures conduisent à des apprentissages. Des chercheurs (Jaffré, 1992, 1995; Fraquet et David, 2013; David et Morin, 2008; Morin, 2005; Morin et Montésinos-Gelet, 2003) se sont penchés sur les conditions à réunir pour que les enfants s'attardent sur ce qu'ils ont écrit en verbalisant des explications à propos de leurs stratégies; ces réflexions 
verbalisées ont été qualifiées d'« explications métagraphiques». Ces explications émises par de jeunes francophones à propos de leurs écritures peuvent témoigner, d'une part, d'une réflexion sur différents aspects de la langue écrite (aspects phonographiques, morphographiques ou encore logographiques) en rendant compte de connaissances et de stratégies orthographiques de plus en plus explicites, et d'autre part, du contrôle cognitif grandissant relativement à la langue écrite, contrôle essentiel au développement d'une expertise orthographique (David et Morin, 2008; Jaffré, 1995; Morin, 2005).

Les enseignants peuvent conduire les élèves à produire des explications métagraphiques en leur posant des questions fréquentes lorsqu'ils écrivent pour les encourager à verbaliser leurs procédures d'écriture et leurs connaissances, souvent encore embryonnaires et non conventionnelles. Ces questions tournent essentiellement autour du pourquoi des choix réalisés et autour du comment de la mise en place des procédures. Des entretiens recueillis par Morin et Montésinos-Gelet (2003) ont montré que le recours à des questions suscitant des explications métagraphiques était très pertinent dans le cadre de l'accompagnement des premières écritures auprès d'enfants de maternelle. Morin (2004) a montré que c'était également le cas auprès de jeunes élèves à risque de vivre des difficultés en écriture au début du primaire. D’une part, ces explications métagraphiques conduisent effectivement les enfants à expliciter leurs stratégies, et d'autre part, elles peuvent servir de point de départ aux échanges entre pairs ou entre élèves et enseignant, et contribuer à la mise en place de conflits sociocognitifs qui eux-mêmes permettront de nouveaux apprentissages.

Adopter un autre rapport à l'erreur pour mieux dégager l'état des connaissances et des stratégies mobilisées par les jeunes scripteurs

Dès la fin des années 1970, Emilia Ferreiro (voir par exemple, Ferreiro et Teberoski, 1982) a attiré l'attention des personnes qui s'intéressaient à l'évolution de la compréhension du fonctionnement de la langue écrite sur le fait que les tentatives d'écriture des enfants évoluaient d'une manière relativement stable d'un enfant à l'autre. À partir d'études et d'observations menées auprès de jeunes hispanophones, elle a établi des stades de développement qui traduisent, selon elle, une découverte progressive et relativement homogène des propriétés de la langue écrite. Aujourd'hui, plusieurs chercheurs s'écartent de cette vision sur la base d'études menées auprès de jeunes scripteurs apprenant des systèmes d'écriture alphabétiques plus ou moins réguliers (en portugais : Alves Martins, Silva et Pereira, 2010; Pollo, Treiman et Kessler, 2008; en anglais : Gentry, 1982, 2005; Read et Treiman, 2013; Treiman et Kessler, 2014; Treiman, Decker, Kessler et Pollo, 2015; en français : Jaffré, 1992; Jaffré et Morin, 2008; David, 2003; Morin, 2007a; comparaison anglais et espagnol : Raynolds et Uhry, 2010). Ils adoptent une vision plus globale du développement orthographique sur la base d'observations qui attirent l'attention sur le fait que les jeunes scripteurs, même s'ils en savent encore peu sur les conventions de la langue écrite, témoignent d'une variété de procédures et de connaissances à l'écrit (Korkeamäki et Dreher, 2000; Rittle-Jonhson \& Siegler, 1999; Sénéchal, 2000) et que celle-ci peuvent être modulées par la nature de la langue à apprendre (par exemple, Pasa et al., 2006). Ces observa- 
tions conduisent même Treiman et Bourassa (2000) à avancer que le développement orthographique serait davantage marqué par une différence de degré plutôt qu’une différence de nature.

Évidemment, pour les jeunes scripteurs confrontés à l'apprentissage d'une langue alphabétique, le défi majeurvers 5-6 ans est celui de la compréhension du principe alphabétique. Toutefois, plusieurs études indiquent que les jeunes enfants élaborent des idées sur l'écrit plus tôt et n'attendent souvent pas de maitriser complètement les règles qui régissent la transcription des phonèmes en graphèmes pour donner sens aux informations morphologiques la plupart du temps inaudibles en français écrit. Cette gestion concomitante de la dimension phonologique et morphologique de l'écriture en français a été notamment soulevée par des études examinant finement les productions écrites de jeunes scripteurs en maternelle et en première année du primaire (Sénéchal, 2000; Morin, 2005), mais aussi par des études ayant plutôt étudié les commentaires - ou explications métagraphiques - émis par des jeunes scripteurs de même âge que l'on invitait à justifier et à expliquer leurs traces écrites (David, 2008).

Montésinos-Gelet et Morin (2006) ont étudié les tentatives d'écriture - lesquelles s'écartent souvent de la norme orthographique attendue - de jeunes enfants et ont élaboré, à partir de ces dernières, une typologie des préoccupations qu'ils entretiennent à l'égard de la langue écrite. Cette typologie attire l'attention sur l'idée que les enfants élaborent très tôt - avant l'enseignement formelle de la langue écrite - des idées sur l'écrit, et que ces dernières constituent la fondation de connaissances sur l'écrit de plus en plus conformes au système écrit qui fait l'objet d'une appropriation progressive.

Cette typologie permet ainsi de concevoir les premières tentatives en écriture non pas comme des « fautes » à éviter, mais plutôt comme des « productions pertinentes » (Jaffré et Morin, 2008) qui correspondent à un passage progressif - voire nécessaire- vers une compréhension plus « conventionnelle » du code écrit. Ainsi, la prise en compte de ces premières tentatives invite à concevoir l'erreur comme étant une trace visible des connaissances acquises et des connaissances en construction chez les enfants afin de les accompagner pour une progression en orthographe. Ce nouveau regard contribue à envisager que le développement des connaissances sur l'écrit est caractérisé par des préoccupations variées dont témoigne le jeune enfant, parfois simultanément, par ses productions et éventuellement les explications qui les accompagnent. Ces préoccupations peuvent être dites visuographiques, phonographiques et orthographiques (Morin, 2007b).

Les préoccupations visuographiques réfèrent à cette attention que l'enfant porte aux aspects visuels des traces qu'il produit: différents aspects du schéma de mise en page (orientation de gauche à droite, du haut vers le bas) et des caractères utilisés (orientation des lettres, la mobilisation des lettres de l'alphabet). Ce type de préoccupations dont peuvent témoigner exclusivement certains enfants suggère que ces derniers distinguent l'écriture du dessin, sans pour autant faire correspondre une valeur sonore aux caractères produits. Les préoccupations phonographiques, qui émergent souvent entre 5 et 6 ans, traduisent une compréhension du principe alphabétique qui sous-tend le français écrit. Ces préoccupations phonographiques conduisent les jeunes scripteurs à établir un lien entre l'oral et 
l'écrit, en témoignant d'une capacité plus ou moins raffinée à analyser les unités de la langue orale (par exemple, un enfant peut produire partiellement les phonèmes du mot « vélo » en écrivant VO, en comparaison avec un autre qui produit ROB pour le mot « robe »). Par ailleurs, pour développer une réelle compétence à l'écrit chez des enfants francophones qui ont à s'approprier un système écrit complexe, ces derniers doivent manifester des préoccupations orthographiques. On peut distinguer deux formes de préoccupations orthographiques. Tout d'abord, certaines de ces préoccupations peuvent être qualifiées de phonographiques dans le sens où elles portent sur les liens qui unissent lettres et sons (la polyvalence des correspondances phonèmes-graphèmes; par exemple, le son /i/ peut se transcrire « $i$ » ou « $y$ »). Ensuite, d'autres peuvent être qualifiées de lexicales en référence au phénomène d'homophonie en français, ou encore dites morphographiques; ces dernières se manifestent, par exemple, par l'attention portée aux indices morphologiques présents dans les mots écrits (mes amis, chat) ou encore aux conventions orthographiques (le mot « manteau » ne s'écrit pas «manto »). En effet, les contacts de plus en plus fréquents et variés avec l'écrit permettent aux enfants de s'interroger et d'attribuer un sens à des unités graphiques qui obéissent à d'autres règles que celles régissant les correspondances graphophonétiques, et dont la fréquence est plus élevée à l'écrit (du moins en français). Grâce à une sensibilité grandissante face aux faits orthographiques à l'écrit, les jeunes scripteurs multiplient leurs connaissances sur les mots et augmentent leur lexique mental.

Dans ce contexte, si l'on adopte la perspective vygotskienne, échanger avec les enfants autour de préoccupations qu'ils verbalisent (pour consolider certaines de leurs découvertes) et attirer leur attention sur des objets d'apprentissage qui sont à leur portée serait une voie d'accompagnement appropriée. Pour illustrer, l'enseignant pourrait attirer l'attention d'un élève qui témoigne de préoccupations visuographiques (par exemple, il produit des vagues pour écrire) sur les symboles spécifiques utilisés en écriture, c'est-à-dire les lettres. Ou encore, l'enseignant pourrait susciter des réflexions sur certains aspects propres à l'orthographe du français (existence de différents graphèmes « eau, au, 0 » pour transcrire un même phonème /o//) pour un autre enfant qui est entré dans la phonétisation de l'écriture. Il faut toutefois veiller à ce que ces éléments restent des repères structurants et permettent à l'enfant de poursuivre ces découvertes sur l'écrit en développant peu à peu une compréhension de plus en plus fine du système écrit. En effet, il peut arriver qu'un enfant qui ne s'intéresse pas encore à l'écrit se pose spontanément des questions d'ordre stratégique (par exemple, comment je peux faire pour retenir comment s'écrit mon prénom ?) Ces questions spontanées peuvent donner lieu à des échanges, quel que soit le niveau de l'enfant. Ainsi, s'appuyer sur les intérêts explicitement manifestés par les enfants et provoquer une réflexion de l'enfant pour des préoccupations qu'il n'a pas encore, mais qu'on sait qu'il devrait avoir prochainement, serait pertinent. 
Ajoutons à cela qu'intervenir de manière appropriée pour accompagner les tentatives d'écriture des enfants pourrait se résumer par une posture simple: d'une part, favoriser des moments d'écriture qui permettent aux enfants de mettre sur papier et de verbaliser ce qu'ils ont compris sur la langue écrite, et d'autre part, questionner, susciter des mises en relation, enrichir la réflexion des enfants de manière à les faire progresser.

\section{Susciter des accompagnements en écriture qui se fondent sur le niveau initial des enfants}

Certaines recherches, encore peu nombreuses, colligent ce sur quoi portent les étayages qui peuvent être mis en place pour accompagner les enfants. Dans ce cadre, une étude réalisée par Pulido, Lacroix et Lainé (2013) fournit des exemples d'étayages offerts par une enseignante de maternelle, en situation d'accompagnement des premières écritures, pour échanger autour du principe alphabétique, de la combinatoire, de la phonologie ou des stratégies à mettre en œuvre pour écrire ou encore pour se rappeler la manière dont s'écrit un mot. Cabell, Tortorelli et Gerde (2013) mettent de l'avant des étayages appropriés selon les préoccupations des enfants. En voilà deux exemples : pour un enfant qui montre des préoccupations visuographiques, l'encourager systématiquement à signer ses travaux; pour un enfant qui montre des préoccupations liées au principe alphabétique, l'aider à identifier le son initial du mot avant de l'aider à écrire. VanNess, Murnen et Bertelsen (2013) partent de leur expérience de la pratique de l'accompagnement des premières écritures et montrent une stratégie de modélisation dans laquelle elles interviennent régulièrement auprès des enfants en écrivant devant eux et en verbalisant à voix haute toutes les stratégies qu'elles utilisent, avec l'objectif que les enfants le fassent aussi en retour. Levin et Aram (2013) s'intéressent également à la modélisation de certaines stratégies. Ils montrent l'intérêt d'une stratégie qui consiste à montrer aux enfants que, pour écrire, on doit segmenter les mots en sons et trouver une lettre par son (l'étude a été réalisée en hébreu). Sipe (2001), quant à lui, présente deux techniques d'étayages utilisables dans le cadre de l'accompagnement des premières écritures. La première sert lorsqu'un enfant tente d'écrire et qu'il s'aperçoit qu'il lui manque certaines connaissances (par exemple, un enfant qui écrit oiseau /so/ et qui dit qu'il ne sait pas écrire [wa]). Elle consiste à proposer à l'enfant de remplacer le son qu'il ne sait pas écrire par un cadre, de manière à pouvoir y revenir un peu plus tard (dans notre exemple, l'enfant écrit/so/). La seconde technique d'étayage proposée consiste tout simplement à prendre en charge les segments qui posent problème à l'enfant. Dans l'exemple donné précédemment pour « oiseau », il s'agirait de dire à l'enfant que [wa] s'écrit avec un /o/ et un /i/.

\section{Susciter des situations d'écriture signifiantes à partir de contextes langagiers variés en maternelle}

Tel qu'évoqué dans l'introduction de cette contribution, les enfants de maternelle essaient spontanément d'écrire des mots, dès lors qu'ils présentent une curiosité pour la langue écrite. Pour stimuler cet intérêt, plusieurs auteurs ont réfléchi à des situations facilitantes. Ainsi, Besse et l'ACLE (2000) ont interrogé des enseignants de maternelle qui ont été formés à encourager l'écriture chez leurs élèves pour déterminer les conditions qui facilitaient l'émergence d'actes d'écriture. Ils ont pu mettre en 
avant que la présence en classe de chevalet avec des feuilles sur lesquelles les enfants sont autorisés à écrire ou d'un tableau blanc accessible pour les élèves étaient autant de conditions favorables.

Au-delà des conditions matérielles, certains moments de classe ont été mis en avant comme particulièrement propices à l'émergence spontanée ou à l'encouragement des tentatives d'écriture des enfants. Les enseignants interrogés par Besse et l'ACLE (2000) ont mis de l'avant trois situations particulières: lors de la communication d'un message à un pair (par exemple, l'établissement d'une correspondance avec une autre classe); lors de moments de dictée à l'adulte, qui peuvent non seulement permettre à l'enseignant de modéliser la manière d'écrire mais peuvent aussi conduire à confier aux enfants l'écriture de quelques mots simples ou déjà connus; ou encore, lors de productions libres de dessins qui, selon les enseignants, constituent des situations où les enfants tentent régulièrement d'écrire spontanément des commentaires.

Charron, Montésinos-Gelet et Morin (2009) ont interrogé cinq enseignantes qui mettaient en place des démarches de soutien des premières écritures des enfants afin de savoir dans quel contexte ce soutien s'avérait le plus propice. Toutes les enseignantes interrogées encourageaient les enfants à écrire lors d'activités de type «mot du jour ». Quatre des cinq enseignantes interrogées profitaient des temps d'arts plastiques pour encourager les élèves à inclure à leurs œuvres quelques mots de présentation ou des commentaires. Parmi les autres contextes évoqués par au moins deux enseignantes comme étant propices pour faire en sorte que les enfants tentent d'écrire un mot, on notera par exemple l'écriture du message du matin ou la création d'un livre.

Montésinos-Gelet et Morin (2006) ont quant à elles identifié différentes situations langagières adaptées à la maternelle, qui sont propices à susciter des tentatives d'écriture, la plupart dans une perspective transversale des apprentissages. Pour illustrer, il est suggéré de plonger les enfants en début de maternelle ( 5 ans) dans l'observation, l'analyse et l'écriture des prénoms des enfants de la classe. Sur ce point, des études ont effectivement montré que, parmi tous les mots que les enfants d'âge préscolaire côtoient dans leur environnement, leur prénom est l'un des premiers qu'ils reconnaissent à l'écrit et qu'ils écrivent de manière conventionnelle (Levin, Both-de Vries, Aram et Bus, 2005; Treiman, Kessler et Polo, 2006). Ces activités à propos des prénoms des enfants de la classe permettent non seulement d'examiner les constituantes linguistiques de ces mots connus et reconnus, le plus souvent globalement, par les enfants, mais aussi de susciter une base de connaissances pour développer différentes procédures et connaissances qui pourront servir de base pour écrire des mots moins connus à l'écrit par les enfants (par exemple, les enfants pourront recourir au début du prénom Antoine pour écrire la fin de maman ou de éléphant). De façon complémentaire, Montésinos-Gelet et Morin (2006) invitent les enseignants à recourir à différentes routines langagières existantes en classe maternelle (le message du matin, le mot du jour, la lecture d'histoires) pour susciter des tentatives d'écriture de mots. 
Dans le but de modéliser les comportements et préoccupations des enfants qui entrent dans l'écrit, Montésinos-Gelet et Morin (2006) suggèrent aussi que l'enseignant suscite des moments de production d'énoncés à l'écrit, notamment par l'entremise de la pratique de la dictée à l'adulte. Cette pratique met en œuvre un contexte où les enfants dictent à l'enseignant le message à écrire et c'est ce dernier qui prend en charge l'écriture proprement dite. Les échanges et les questions de l'enseignant permettent ainsi une négociation à propos de la formulation écrite qui pourra notamment porter sur les choix lexicaux, l'orthographe des mots, la segmentation des énoncés (emplacement des blancs graphiques) (Chartier, Clesse et Hébrard, 1998; David, 1991). Plus concrètement, l'enseignant pourrait profiter du temps généralement consacré, en début d'année scolaire, à l'explicitation des règles de vie de la classe, pour inciter les enfants à écrire. Pour cela, après avoir échangé autour des règles de vie de la classe, l'enseignant entreprend de les écrire. Il écrit les premières en explicitant comment il s'y prend, puis propose aux enfants d'intervenir directement dans l'écriture des dernières en situation collective. Cette initiation à l'écriture d'idées peut être non seulement valorisée en sollicitant la dictée à l'adulte, mais aussi réalisée par écriture autonome de la part des élèves, ce qui permet, dans ce cas, à l'enseignant de situer la capacité des enfants à s'approcher de l'écriture d'une phrase (par exemple, les enfants introduisent-ils des blancs graphiques? Recourent-ils partiellement à un traitement phonologique pour écrire des mots ?). La figure qui suit illustre la production d'une phrase par un enfant en fin de maternelle.

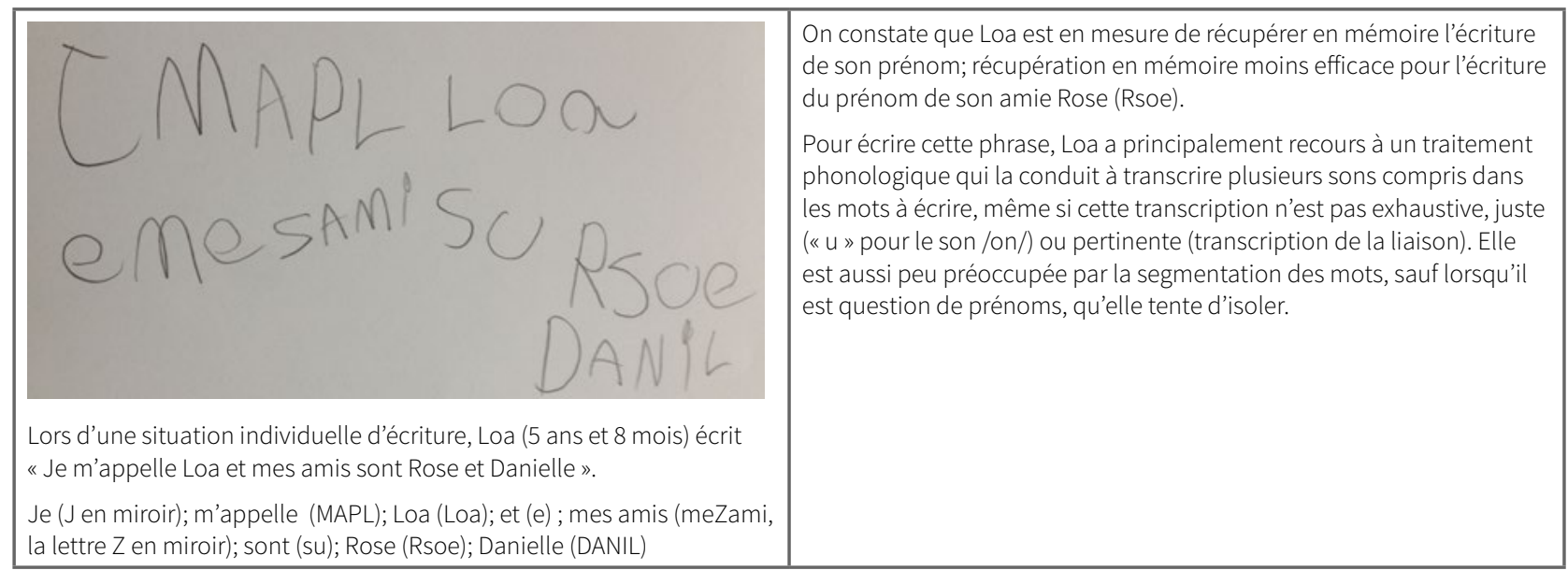

\section{Figure 1 : Exemple de production d'un énoncé en situation libre d'écriture}

\section{Une démarche didactique : les orthographes approchées}

Dans un ouvrage à portée didactique, Montésinos-Gelet et Morin (2006) font état de préconisations auxquelles elles ont pu aboutir grâce à un projet de recherche qui interrogeait les conditions dans lesquelles le soutien des premières écritures constitue une démarche d'entrée dans l'écrit. La démarche proposée formalise une succession d'étapes pour conduire les élèves à tenter d'écrire des mots pour approcher l'orthographe. Cette démarche induit un travail avec de petits groupes d'élèves et se compose de phases différentes dont le point de départ est ancré dans les capacités indivi- 
duelles à faire une première tentative en écriture pour aboutir à la norme orthographique par des interactions entre pairs et avec l'enseignant.

Cette démarche peut être ainsi décomposée:

- Écriture individuelle d'un mot: ce mot (ou ce groupe de mots) est choisi par les enfants ou l'enseignant à partir d'un contexte signifiant de communication écrite pour les enfants (lecture d'une histoire, message à transmettre à autrui, production artistique, etc.).

- Partage d'hypothèses en équipe (binôme ou trio) recherche d'un consensus; cette seconde phase permet aux enfants de mettre en commun leurs propositions individuelles et surtout d'accéder à une nouvelle proposition commune qui fera consensus sur la base d'arguments de plus en plus explicites. Ce travail en équipe peut par exemple être facilité en répartissant des rôles entre les enfants (par exemple, un scripteur, un responsable d'un alphabet qui peut servir d'aide-mémoire, un responsable de la gomme, un responsable du temps); au cours de cette phase de réflexion sur l'écriture du mot, l'enseignant modélise le questionnement et les échanges en encourageant le petit groupe d'enfants avec lequel il travaille à utiliser leurs connaissances et à verbaliser leurs stratégies. II agit également à titre de guide, en prenant en compte les préoccupations des élèves, pour les amener à faire des découvertes sur le fonctionnement de la langue écrite.

- Retour en groupe-classe sur les propositions orthographiques. Durant cette phase, l'enseignant valorise le travail du groupe auprès de l'ensemble de la classe en laissant les élèves présenter leur réflexion ou en affichant l'écriture à laquelle ils sont parvenus. L'idée de cette phase est de pouvoir partager les avancées des élèves et les stratégies adoptées lorsque celles-ci sont réalisables.

- Enfin, une dernière phase consiste à rechercher la norme orthographique pour le mot cible. II s'agit d'envisager avec les enfants les avenues possibles pour en arriver à l'orthographe du mot et, le cas échéant, à faire réfléchir les enfants à l'écart avec leur proposition. La norme orthographique trouvée pourra être consignée afin, ultérieurement, que les enfants puissent y recourir dans un autre contexte d'écriture de ce même mot ou dans un contexte où ce mot pourra servir de base pour en écrire un nouveau ( «j'écris la fin de chaton comme dans la fin du mot mouton que nous avons écrit la semaine dernière ».) Les découvertes des enfants en écriture étant variées, les réinvestissements le sont également. En effet, il peut s'agir d'essayer de se souvenir de l'écriture d'un mot cible pour lequel l'orthographe a été stabilisée, ou encore de réemployer une stratégie qui a été utilisée et partagée en groupe (consulter un livre ou un affichage dans la classe pour se servir d'un mot ou d'une partie de mot pour écrire). 


\section{Quelques illustrations de démarches d'accompagnement des premières écritures}

Deux équipes de recherches ont développé des outils qui facilitent l'émergence des tentatives d'écriture des enfants et leur accompagnement (Richgels, 2013; Pulido et al., 2014).

Plus précisément, Richgels (2013) a élaboré un support qui permet d'intégrer des tentatives d'écriture dans une démarche globale d'éveil à l'écrit. Cette démarche initiée auprès de jeunes anglophones se nomme Talk, Write \& Read (TWR) et vise à proposer une routine langagière favorisant la découverte de la lecture et de l'écriture sur la base d'échanges à l'oral.

En s'appuyant sur un support illustré appelé «From far away and up close », l'enseignant fait décrire des images à l'oral aux élèves. Les images sont présentées par paires : un plan large et un gros plan d'une partie de l'image en plan large. Le gros plan est accompagné d'une phrase inductrice lue par l'enseignant: «Dans la photo prise de loin, je vois beaucoup de choses. Dans celle prise de près, je vois... . À partir de là, les élèves doivent compléter la phrase, par écrit, avec les lettres d'un alphabet mobile. L'objectif de ce dispositif est de conduire les enfants à écrire une série de mots simples, soutenus par leur enseignant: car, boat, sign, bus, duck, bench, flag et train.

De leur côté, Pulido et al. (2014) ont développé un jeu d’orthographes approchées : Grapho Logic. Ce jeu de chasse aux trésors a été développé dans une démarche collaborative chercheurs / conseillers pédagogiques / enseignants. Les enfants jouent par équipe de trois. Ils doivent collaborer pour écrire des mots présentés sur des images. Une collaboration efficace permet d'accéder à une clé qui déverrouille un coffre aux trésors.

Pour chaque mot, l'enseignant aide :

1. chaque enfant à écrire individuellement avec des lettres mobiles;

2. chaque enfant à présenter sa proposition et ses stratégies d'écriture;

3. les trois élèves de l'équipe à discuter pour arriver à une proposition collective;

4. à confronter la proposition à la norme orthographique et à réfléchir aux éventuels écarts entre les deux écritures.

Le jeu propose 60 mots présentant des caractéristiques variées, de difficultés orthographiques progressives. D’abord, les enfants réfléchissent à des mots de deux ou trois syllabes composées de deux sons. Chaque son s'écrit avec une lettre (par exemple, moto). Cette catégorie vise à échanger autour des lettres, du principe alphabétique, des stratégies de segmentation pour des mots présentant une structure phonologique simple. Ensuite, les enfants réfléchissent à des mots présentant les caractéristiques de la catégorie précédente, mais dont un son s'écrit avec un bigramme ou un trigramme (par exemple, lapin). Ces mots permettent de renforcer les apprentissages réalisés avec la première liste et permettent d'échanger sur le statut des bigrammes et trigrammes, voire d'en ap- 
prendre quelques-uns. Dans un troisième temps, les enfants sont confrontés à des mots équivalents aux précédents mais contenant au moins une syllabe que l'on peut décomposer en trois phonèmes (par exemple, avion). Ces mots permettent d'échanger sur les stratégies à avoir pour encoder des structures syllabiques un peu plus complexes. Enfin, dans un quatrième temps, les mots proposés contiennent des segments dont l'orthographe est peu fréquente (par exemple, oignon). Ces mots permettent aux enfants de découvrir que pour certains mots, l'orthographe ne peut pas être devinée. Ils permettent parfois de réfléchir à des stratégies à mettre en place pour tenter de se souvenir d'un mot.

\section{Synthèse : comment accompagner les premières écritures des enfants de maternelle?}

Les recherches dontil a été question dans cette section permettent de dégager des actions concrètes à mettre en œuvre au quotidien par qui souhaite accompagner les premières écritures des enfants: (1) encourager les enfants à tenter d'écrire des mots, en verbalisant leurs stratégies, (2) apprendre à voir dans les tentatives d'écriture des enfants des marques de compréhension du fonctionnement de l'écrit, (3) être sensible aux préoccupations que les enfants entretiennent à l'égard de la langue écrite, et (4) tirer parti de contextes variés pour faire écrire les élèves.

Au-delà de ces actions quotidiennes, les recherches recensées permettent d'avoir des pistes d'actions concrètes pour accompagner les élèves. Ces pistes sont de deux ordres : une démarche didactique pour faire écrire les élèves (la démarche des orthographes approchées); des activités concrètes calibrées pour mettre en place une telle démarche.

\section{Conclusions}

La première section de ce chapitre permet de comprendre, à la lumière de résultats obtenus par des études empiriques, l'intérêt pour les élèves d'être accompagnés lorsqu'ils réalisent leurs premières tentatives d'écriture. En effet, les études recensées montrent des effets positifs de l'accompagnement des premières écritures en maternelle sur différentes compétences qui constituent de bons prédicteurs d'un apprentissage aisé de la lecture et de l'écriture.

Les résultats actuels de la recherche encouragent la mise en place de dispositifs variés d'écriture en maternelle. D'une part, des dispositifs qui encouragent les jeunes enfants à écrire dans différentes situations en classe de maternelle, afin que l'enseignant puisse saisir de la façon la plus juste possible l'état des connaissances des enfants. D'autre part, des dispositifs d'accompagnement des premières écritures qui permettent à l'enseignant de soutenir la progression des enfants quant au développement de différentes capacités soutenant leur appropriation de la langue écrite (capacité phonologique, orthographique et lecture). Ces préconisations insistent sur l'importance de mettre en place des contextes permettant aux enfants de parler de leurs stratégies et d'échanger à propos 
de ces dernières, tout en permettant de fournir aux enfants des aides variées et appropriées au regard de leur niveau et de leurs préoccupations. 


\section{Bibliographie}

Alves Martins, M., Albuquerque, A., Salvador, L. et Silva, C. (2013). The impact of invented spelling on early spelling and reading. Journal of Writing Research, 5, 215-237. doi:10.17239/jowr-2013.05.02.3

Alves Martins, M., Salvador, L., Albuquerque, A. et Silva, C. (2016). Invented spelling activities in small groups and early spelling and reading. Educational Psychology: An International Journal of Experimental Educational Psychology, 36, 738-752. doi:10.1080/01443410.2014.950947

Alves Martins, M. et Silva, C. (2006). The impact of invented spelling on phonemic awareness. Learning and Instruction, 16, 41-56. doi:10.1016/j.learninstruc.2005.12.005

Alves Martins, M., Silva, C. et Pereira, M. (2010). The impact of the articulatory properties of phonemes on the evolution of preschool children's writing. Applied Psycholinguistics, 31(4), 693-709.

Besse, J.-M. et A.C.L.E. (2000). Regarde comme j'écris, écrits d'élèves, regards d'enseignants. Paris : Magnard.

Cabell, S., Tortorelli, L. et Gerde, H. (2013). How Do I Write...? Scaffolding Preschoolers' Early Writing Skills. The reading teacher, 66, 650-659.

Charron, A., Montésinos-Gelet, I. et Morin, M.-F. (2008). La temporalité didactique dans les pratiques déclarées en orthographes approchées chez des enseignantes de maternelle. Revue française de pédagogie, 163, 91-103.

Charron, A. Montésinos-Gelet, I. et Morin, M.-F. (2009). Description et catégorisation des pratiques déclarées en orthographes approchées chez des enseignantes du préscolaire. Revue des sciences de l'éducation, 35(3), 85-106.

Chartier A.-M., Clesse, C. et Hébrard, J. (1998). Lire, écrire 2. Produire des textes au cycle 2. Paris : Hatier.

Chomsky, C. (1971). Write first, read later. Childhood Education, 47, 296-300.

David, J. (1991). La dictée à l'adulte ou comment de jeunes enfants oralisent l'écrit... Études de linguistiques appliquées, 81, 7-19.

David, J. (2003). Les procédures orthographiques dans les productions écrites des jeunes enfants. Revue des sciences de l'éducation, XXIX(1), 137-158.

David, J. (2008). Les explications métagraphiques appliquées aux premières écritures enfantines. Pratiques, 139/140, 163-187.

David, J. et Morin, M.-F. (2008). Écritures approchées : des procédures métagraphiques des jeunes apprentis-scripteurs aux pratiques d'apprentissage. Dans J. Dolz et S. Plane (dir.), Formation des enseignants et enseignement de la lecture et de l'écriture. Recherches sur les pratiques (p. 19-41). Namur: Presses Universitaires de Namur.

Fayol, M. et Jaffré, J.-P. (2014). L'orthographe. Paris : Presses Universitaires de France. 
Ferreiro, E. et Teberosky, A. (1982). Literacy before schooling. Portsmooth : Heinemann Educational Book.

Fijalkow, J., Cussac-Pomel, J. et Hannouz, D. (2009). L'écriture inventée : empirisme, constructivisme, socioconstructivisme, Éducation et didactique, 3(3), mis en ligne le 01 octobre 2011, consulté le 28 janvier 2016. URL : http://educationdidactique.revues.org/576

Fraquet, S. et David, J. (2013). Écrire en maternelle : comment approcher le système écrit? Repères, 47, 19-40.

Gentry, J. R. (1982). An analysis of developmental spelling in GNYS AT WRK. The reading teacher, 36, $192-200$.

Gentry, J. R. (2005). Instructional techniques for emerging writers and special needs students at kindergarten and grade 1 levels. Reading \& Writing Quarterly, 21(2), 113-134.

Goswami, U. et Bryant, P. (1990). Phonological Skills and Learning to Read. New-York : Psychology Press.

Hofslundsengen, H., Hagtvet, B. et Gustafsson, J.-E. (2016). Immediate and delayed effects of invented writing intervention in preschool. Reading and writing, 29(7). DOI 10.1007/s11145-016-9646-8

Jaffré, J. P. (1992). Le traitement élémentaire de l'orthographe : les procédures graphiques. Langue Française, 95, 27-48.

Jaffré, J. P. (1995). Compétence orthographique et acquisition. Dans D. Ducard, R. Honvault et J. P. Jaffré, (dir.), L'orthographe en trois dimensions (p. 94-158). Paris : Nathan.

Jaffré, J.-P. et Morin, M.-F. (2008). Les activités pré-orthographiques : nature, validité et conceptions. Pratiques, 139/140, 189-207.

Korkeamäki, R. L. et Dreher, M. J. (2000). Finnish kindergartners' literacy development in contextualized literacy episodes: A focus on spelling. Journal of Literacy Research, 32(3), 349-393.

Levin, I. et Aram, D. (2013). Promoting Early Literacy via Practicing Invented Spelling: A Comparison of Different Mediation Routines. Reading research Quarterly, 48(3), 221-236.

Levin, I., Both-de Vries, A., Aram, D. et Bus, A. (2005). Writing starts with own name writing: From scribbling to conventional spelling in Israeli and Dutch children. Applied Psycholinguistics, 26, 463-477

MELS (2001). Programme de formation de l'école québécoise. Disponible à : http://www1.mels.gouv. qc.ca/sections/programmeFormation/pdf/prform2001.pdf (consulté le 15 mars 2016).

MEN (2015). Programme d'enseignement de l'école maternelle. Disponible à : http://www.education. gouv.fr/pid25535/bulletin_officiel.html?cid_bo=86940 (consulté le 15 mars 2016).

Montésinos-Gelet, I. et Besse, J.-M. (2003). La séquentialité phonogrammique en production d’orthographes inventées. Revue des Sciences de l'Éducation, XXIX(1), 159-170. 
Montésinos-Gelet, I. et Morin, M. F. (2005). The impact of a cooperative approximate spelling situation in a kindergarten setting. L1-Education Studies in Language and Literature, 5, 365-383.

Montésinos-Gelet, I. et Morin, M.-F. (2006). Les orthographes approchées. Montréal : Chenelière éducation.

Morin, M.-F. (2004). Comprendre et prévenir les difficultés en écriture chez le jeune enfant en examinant les orthographes approchées et les commentaires métagraphiques. Dans J.-C. Kalubi et G. Debeurme (dir.), Identités professionnelles et interventions scolaires. Contextes de formation de futurs enseignants (p.145-173). Sherbrooke : Éditions du CRP.

Morin, M.-F. (2005). Declared Knowledge of Beginning Writers. L1-Educational Studies in Language and Literature, 5(3), 385-401.

Morin, M.-F. (2007a). Linguistic factors and invented spelling in children: The case of learning writing in Canada. L1-Educational Studies in Language and Literature, 7(3), 173-189.

Morin, M.-F. (2007b). Une vision globale de l'appropriation de l'écrit en maternelle. Dans A.-M. Dionne (dir.) et M.-J. Berger (dir.), Les littératies : perspectives linguistique, familiale et culturelle (p.7-34). Ottawa: Les Presses de l'Université d'Ottawa.

Morin, M.-F. et Montésinos-Gelet, I. (2003). Les commentaires métagraphiques en situation collaborative d'écriture chez les enfants de maternelle. Archives de psychologie, 70, 41-65.

Morin, M.-F. et Montésinos-Gelet, I. (2007). Effet d'un programme d'orthographes approchées en maternelle sur les performances ultérieures en lecture et en écriture d'élèves à risque. Revue des Sciences de l'Education, 33, 663683. doi:10.7202/018963ar

Noyer-Martin, M. et Baldy, R. (2008). L'acquisition de l'écriture chez les enfants de 3 à 8 ans : approche évolutive transverse. Bulletin de Psychologie, 5(497), 449-459.

O'connor, R., \& Jenkins, J. (1995). Improving the generalization of sound/symbol knowledge: teaching spelling to kindergarten children with disabilities. The journal of special education, 29(3), 255-275.

Ouellette, G. et Sénéchal, M. (2008). Pathways to literacy: A study of invented spelling and its role in learning to read. Child Development, 79, 799813. doi:10.1007/s11145-014-9517-0

Ouellette, G., Sénéchal, M. et Haley, A. (2013). Guiding children's invented spellings: A gateway into literacy learning. Journal of Experimental Education, 81, 261279. doi:10.1080/00220973.2012.699 903

Pasa, L., Creuzet, V. et Fijalkow, J. (2006). Écriture inventée : pluralité des traitements et variabilité selon la structure syllabique. Éducation et Francophonie, 34(2), 85-103.

Pollo, T., Treiman, R. et Kessler, B. (2008). Preschoolers use partial letter names to select spellings: Evidence from Portuguese. Applied Psycholinguistics, 29, 195-212. doi: 10.1017/S0142716407080095

Pulido, L., Lacroix, F. et Lainé, A. (2013). Interaction en classe, dans des situations d'orthographes approchées. Repères, 47, 41-57. 
Pulido, L., Lacroix, F., Lainé, A., Carette, M. A., Colnet, D., Jourdet, S. et Perrin, V. (2014). Grapho-logic, ateliers d'orthographes approchées pour la maternelle. Grenoble : éditions la Cigale.

Raynolds, L. B. et Uhry, J. K. (2010). The invented spellings of non-Spanish phonemes by SpanishEnglish bilingual and English monolingual kindergarteners. Reading and Writing, 23, 495-513.

Read, C. (1971). Pre-school children's knowledge of English phonology. Harvard Educational review, 41, 1-134.

Read, C. et Treiman, R. (2013). Children's invented spelling: What we have learned in forty years. Dans M. Piattelli-Palmarini et R. C. Berwick (dir.), Rich languages from poor inputs (p.197-211). New York: Oxford University Press.

Richgels, D. (2013). Talk, Write, and Read: A Method for Sampling Emergent Literacy Skills. The Reading Teacher, 66(5), 380-389.

Rieben, L., Ntamakiliro, L., Gonthier, B. et Fayol, M. (2005). Effects of various early writing practices on reading and spelling. Scientific Studies of Reading, 9, 145166. doi:10.1207/s1532799xssr0902_3

Rittle-Jonhson, B. et Siegler, R. S. (1999). Learning to spell: Variability, choice, and change in children's strategy use. Child Development, 70(2), 332-348.

Santoro, L., Coyne, M. et Simmons, D. (2008). The Reading-Spelling Connection: Developing and Evaluating a Beginning Spelling Intervention for Children at Risk of Reading Disability. Learning Disability practice, 21(2), 122-133.

Sénéchal, M. (2000). Morphological effects in children's spelling of French words. Canadian Journal of Experimental Psychology, 54(2), 76-85.

Sénéchal, M., Ouellette, G., Pagan, S. et Lever, R. (2012). The role of invented spelling on learning to read in low-phoneme-awareness kindergartners: A randomized-control-trial study. Reading and Writing, 4, 917934. doi:10.1007/s11145-011-9310-2

Sipe, L. (2001). Invention, convention, and intervention: invented spelling and the teacher's role: this article highlights the teacher's critical role in spelling instruction and provides examples of how to support spelling development in classrooms. The reading teacher, 55(3), 264-273.

Treiman, R. et Bourassa, D. (2000). Children's written and oral spelling. Applied Psycholinguistics, 21, 183-204.

Treiman, R., Decker, K., Kessler, B. et Pollo, T. C. (2015). Variation and repetition in the spelling of young children. Journal of Experimental Child Psychology, 132, 99-110.

Treiman, R. et Kessler, B. (2014). How children learn to write words. New York, NY: Oxford University Press.

Treiman, R., Kessler, B. et Pollo, T. C. (2006). Learning about the letter name subset of the vocabulary: Evidence from U.S. and Brazilian preschoolers. Applied Psycholinguistics, 27, 211-227. doi: $10.1017 /$ S0142716406060255 
VanNess, A., Murnen, T. et Bertelsen, D. (2013). Let me tell you a secret: kindergartners can write. The reading teacher, 66, 574-585.

Vasconcelos Horta, I. \& Alves Martins, M. (2011). Invented spelling programmes and the access to the alphabetic principle in kindergarten. LI Educational Studies in Language and Literature, 11, 1-23. 